\title{
Symmetry of Brans-Dicke gravity as a novel solution-generating technique
}

\author{
Valerio Faraoni,, , * Dilek K. Çiftci, ${ }^{2,1, \text {, }}$ and Shawn D. Belknap-Keet ${ }^{3, \text { I }}$ \\ ${ }^{1}$ Department of Physics and Astronomy and STAR Research Cluster, \\ Bishop's University, 2600 College Street, Sherbrooke, Québec, Canada J1M 1Z7 \\ ${ }^{2}$ Department of Physics, Namık Kemal University, Tekirdă̆, Turkey \\ ${ }^{3}$ Department of Physics and Astronomy, Bishop's University, \\ 2600 College Street, Sherbrooke, Québec, Canada J1M 1Z7
}

\begin{abstract}
A symmetry of Brans-Dicke gravity in (electro)vacuo or in the presence of conformally invariant matter is presented and used as a solution-generating technique starting from a known solution as a seed. This novel technique is applied to generate, as examples, new spatially homogeneous and isotropic cosmologies, a 3-parameter family of spherical time-dependent spacetimes conformal to a Campanelli-Lousto geometry, and a family of cylindrically symmetric geometries.
\end{abstract}

\section{INTRODUCTION}

There is plenty of motivation for studying theories of gravity alternative to General Relativity (GR), both theoretically and experimentally. Attempts to quantize GR invariably introduce modifications to it in the form of extra dynamical fields or higher order field equations, and these corrections are not necessarily Planck-scale suppressed. The prototype of the alternative to GR is scalar-tensor gravity. Its simplest incarnation is BransDicke theory [1], which was generalized to richer forms of scalar-tensor gravity [2]. In the 1980s, waning interest in this class of theories by the gravity community was renewed by the realization that the simplest string theory, bosonic string theory, reduces to $\omega=-1$ Brans-Dicke gravity in the low-energy limit [3].

More urgent motivation comes from cosmology. The 1998 discovery that the expansion of the universe is accelerated can be explained by the standard $\Lambda$ cold dark matter cosmological model based on GR only at the price of introducing a completely ad hoc dark energy accounting for approximately $70 \%$ of the energy content of the universe [4]. A possible way to avoid introducing dark energy is by modifying gravity. Many theories of modified gravity have been studied and intense experimental and theoretical efforts aiming at testing gravity are underway or under planning (see the reviews [5]). Probably the most popular class of modified gravity theories motivated by cosmology is $f(R)$ gravity ([6], see [7] for reviews). $f(R)$ gravity turns out to be a Brans-Dicke theory in disguise, corresponding to the special value $\omega=0$ of the Brans-Dicke coupling and to a special potential for the scalar degree of freedom [7]. Apart from $f(R)$ gravity, Brans-Dicke theory is the toy model of choice to explore deviations from GR involving scalar degrees of freedom in many areas, including cosmology, black holes, gravitational waves, no-hair theorems and ways to evade them, stealth fields, and apparent horizons. Older re-

\footnotetext{
*vfaraoni@ubishops.ca

$\dagger$ dkazici@ubishops.ca, dkazici@nku.edu.tr

‡ sbelknapkeet02@ubishops.ca
}

search which led to the introduction of the original BransDicke theory involves Mach's principle [1] and Dirac's idea that the constants of nature may actually be dynamical fields [8], which is partially realized in the feature of Brans-Dicke gravity that the effective gravitational coupling strength becomes, roughly speaking, the inverse of the Brans-Dicke scalar field $\phi$ [1]. There has been renewed interest in varying "constants" of physics in recent years (see [9] for a popular exposition). Extra motivation related to the quantization of gravity is provided by the finding that generalized Brans-Dicke solutions describe asymptotically Lifshitz black holes [10].

Analytical solutions of scalar-tensor gravity can provide insight into aspects of these directions of research, but they are not as numerous as the better known solutions of GR [11]. It is valuable, therefore, to find general solution-generating techniques in scalar-tensor gravity. Here we focus on a symmetry group of Brans-Dicke gravity (enriched by the possibility of an arbitrary potential $V(\phi)$ for the Brans-Dicke scalar field) in the presence of conformally invariant matter [12], which is really a restricted conformal invariance of the theory and is reminiscent of the broader conformal invariance of string theories [13]. We explore the use of this symmetry as a novel technique to generate new solutions of Brans-Dicke gravity using known solutions as seeds. As examples of application of this technique, we find three different kinds of analytical solutions: in the cosmological context, then spherically symmetric and time-dependent solutions, and finally cylindrically symmetric geometries. We use units in which the speed of light in vacuo and Newton's constant are unity and we follow the notation of Ref. [14].

\section{A SYMMETRY OF BRANS-DICKE THEORY}

In this section we generalize the symmetry of BransDicke theory with $V(\phi)=0$ found in 12] to the case in which the Brans-Dicke scalar field $\phi$ is endowed with a potential and conformally invariant matter is present. For ease of exposition, we begin with the vacuum theory and, in the last subsection, we include conformally invariant matter. 


\section{A. Vacuum Brans-Dicke theory with any potential}

The action is

$$
S_{B D}=\int d^{4} x \sqrt{-g}\left[\phi R-\frac{\omega}{\phi} g^{a b} \nabla_{a} \phi \nabla_{b} \phi-V(\phi)\right] .
$$

This action is invariant in form under the operation $\left(g_{a b}, \phi\right) \rightarrow\left(\tilde{g}_{a b}, \tilde{\phi}\right)$, where

$$
\begin{aligned}
\tilde{g}_{a b} & =\Omega^{2} g_{a b}=\phi^{2 \alpha} g_{a b}, \\
\tilde{\phi} & =\phi^{1-2 \alpha},
\end{aligned}
$$

for $\alpha \neq 1 / 2$, that is, a conformal transformation of the metric with conformal factor $\Omega=\phi^{\alpha}$ and a non-linear redefinition of the scalar field. Since it is $\phi>0$ to guarantee the positivity of the gravitational coupling, the conformal transformation is well defined (except at spacetime points where $\phi$ diverges, which are to be regarded as physical singularities). A tilde denotes geometric quantities constructed with the conformally rescaled metric $\tilde{g}_{a b}$. By using the well-known transformation properties [14 17$]$

$$
\begin{aligned}
\tilde{g}^{a b} & =\Omega^{-2} g^{a b}, \\
\sqrt{-\tilde{g}} & =\Omega^{4} \sqrt{-g}, \\
\tilde{R} & =\Omega^{-2}\left(R-\frac{6 \square \Omega}{\Omega}\right),
\end{aligned}
$$

and Eq. (2.3), one obtains

$$
\begin{aligned}
R= & \phi^{2 \alpha} \tilde{R}-\frac{6 \alpha(1-\alpha)}{(1-2 \alpha)^{2}} \phi^{6 \alpha-2} \tilde{g}^{a b} \tilde{\nabla}_{a} \tilde{\phi}_{\bar{\nabla}} \tilde{\phi} \\
& +\frac{6 \alpha}{1-2 \alpha} \phi^{4 \alpha-1} \tilde{\square} \tilde{\phi} .
\end{aligned}
$$

The term proportional to $\tilde{\square} \tilde{\phi}$ which appears in the action (as a contribution coming from $\sqrt{-g} \phi R$ ) because of the last term in the right-hand side of Eq. (2.7) can be written as

$$
\frac{6 \alpha}{1-2 \alpha} \sqrt{-\tilde{g}} \tilde{\square} \tilde{\phi}=\frac{6 \alpha}{1-2 \alpha} \partial_{\mu}\left(\sqrt{-\tilde{g}} \tilde{g}^{\mu \nu} \partial_{\nu} \tilde{\phi}\right)
$$

which is integrated to produce a boundary term giving zero contribution when the action is varied. This term is ignored in the following. The Brans-Dicke action (2.1), therefore, becomes

$$
S_{B D}=\int d^{4} x \sqrt{-\tilde{g}}\left\{\tilde{\phi} \tilde{R}-\left[\frac{\omega}{(1-2 \alpha)^{2}}+\frac{6 \alpha(1-\alpha)}{(1-2 \alpha)^{2}}\right] \frac{\tilde{g}^{a b}}{\tilde{\phi}} \tilde{\nabla}_{a} \tilde{\phi}^{\tilde{\nabla}}{ }_{b} \tilde{\phi}-\tilde{\phi}^{\frac{-4 \alpha}{1-2 \alpha}} V(\phi)\right\}
$$

By redefining the Brans-Dicke coupling and scalar field potential as

$$
\begin{aligned}
\tilde{\omega}(\omega, \alpha) & =\frac{\omega+6 \alpha(1-\alpha)}{(1-2 \alpha)^{2}}, \\
\tilde{V}(\tilde{\phi}) & =\tilde{\phi}^{\frac{-4 \alpha}{1-2 \alpha}} V\left(\tilde{\phi}^{\frac{1}{1-2 \alpha}}\right),
\end{aligned}
$$

the Brans-Dicke action is rewritten as [12]

$$
S_{B D}=\int d^{4} x \sqrt{-\tilde{g}}\left[\tilde{\phi} \tilde{R}-\frac{\tilde{\omega}}{\tilde{\phi}} \tilde{g}^{a b} \tilde{\nabla}_{a} \tilde{\phi} \tilde{\nabla}_{b} \tilde{\phi}-\tilde{V}(\tilde{\phi})\right]
$$

i.e., it is invariant in form under the transformation (2.2), (2.3), provided that the changes (2.10), (2.11) are made. In addition, the transformations of the type (2.2), (2.3) form a 1-parameter Abelian group [12].

As a special case, we note that a power-law potential

$$
V(\phi)=V_{0} \phi^{n}
$$

(where $V_{0}$ and $n$ are constants) is invariant in form, i.e., the symmetry produces another power-law potential

$$
\tilde{V}(\tilde{\phi})=V_{0} \tilde{\phi}^{\tilde{n}}
$$

with the new power

$$
\tilde{n}=\frac{n-4 \alpha}{1-2 \alpha}
$$

An even more special case is $n=2$, for which also the power in the potential is left invariant, $\tilde{V}(\tilde{\phi})=m^{2} \tilde{\phi}^{2} / 2=$ $V(\phi)$ and $\tilde{n}=n=2$ when $V(\phi)=m^{2} \phi^{2} / 2$. 


\section{B. Electrovacuum Brans-Dicke theory}

When an electromagnetic field is present as a form of matter, the action is

$$
\begin{aligned}
S_{B D}= & \int d^{4} x \sqrt{-g}\left[\phi R-\frac{\omega}{\phi} g^{a b} \nabla_{a} \phi \nabla_{b} \phi-V(\phi)\right. \\
& \left.-F^{a b} F_{a b}\right]
\end{aligned}
$$

where $F^{a b}$ is the Maxwell tensor. Since the latter has conformal weight $s=0$ [14], $\tilde{F}_{a b}=F_{a b}$ and

$$
\sqrt{-g} F^{a b} F_{a b}=\sqrt{-\tilde{g}} \widehat{F^{a b} F_{a b}}
$$

so that also $\sqrt{-g} \mathcal{L}_{(m)}$ for this form of matter remains invariant under the transformation (2.2), (2.3).

\section{Conformally invariant matter}

It is tempting to ask whether Brans-Dicke theory is left invariant by the transformation (2.2), (2.3) in the presence of any other form of matter, for example conformally invariant matter. This property would be especially important for applications, e.g., in cosmology or in stars when a radiation fluid is present. The action principle for fluids is notoriously nontrivial [18 20], therefore in this case it is more convenient to analyze directly the transformation of the field equations. The variation of the Brans-Dicke action (2.1) with the addition of a matter action produces the field equations

$$
\begin{aligned}
R_{a b}-\frac{1}{2} g_{a b} R & =\frac{8 \pi}{\phi} T_{a b}+\frac{\omega}{\phi^{2}}\left(\nabla_{a} \phi \nabla_{b} \phi-\frac{1}{2} g_{a b} g^{c d} \nabla_{c} \phi \nabla_{d} \phi\right)+\frac{1}{\phi}\left(\nabla_{a} \nabla_{b} \phi-g_{a b} \square \phi\right)-\frac{V}{2 \phi} g_{a b}, \\
\square \phi & =\frac{1}{2 \omega+3}\left[\frac{8 \pi T}{\phi}+\phi \frac{d V}{d \phi}-2 V\right],
\end{aligned}
$$

where $T_{a b}$ is the matter stress-energy tensor and $T$ is its trace. A rather long but straightforward calculation gives the transformation properties of Eqs. (2.18) and (2.19) under the operation (2.2), (2.3). The scalar field equation (2.19) becomes

$$
\tilde{\square} \tilde{\phi}=\frac{1}{2 \tilde{\omega}+3}\left[\frac{8 \pi}{1-2 \alpha} \tilde{\phi}^{\frac{-4 \alpha}{1-2 \alpha}} T+\tilde{\phi} \frac{d \tilde{V}}{d \tilde{\phi}}-2 \tilde{V}\right],
$$

where $\tilde{\omega}$ and $\tilde{V}(\tilde{\phi})$ are given by Eqs. (2.10) and (2.11). Therefore, Eq. (2.19) is invariant in form under the transformation (2.2), (2.3) only for conformally invariant matter with $T=0$.

Under the same transformation, the other field equation (2.18) becomes

$$
\begin{aligned}
& \tilde{R}_{a b}-\frac{1}{2} \tilde{g}_{a b} \tilde{R}=\frac{8 \pi}{\tilde{\phi}^{\frac{1}{1-2 \alpha}}} T_{a b} \\
& +\frac{\tilde{\omega}}{\tilde{\phi}^{2}}\left(\tilde{\nabla}_{a} \tilde{\phi} \tilde{\nabla}_{b} \tilde{\phi}-\frac{1}{2} \tilde{g}_{a b} \tilde{g}^{c d} \tilde{\nabla}_{c} \tilde{\phi} \tilde{\nabla}_{d} \tilde{\phi}\right) \\
& +\frac{1}{\tilde{\phi}}\left(\tilde{\nabla}_{a} \tilde{\nabla}_{b} \tilde{\phi}-\tilde{g}_{a b} \tilde{\square} \tilde{\phi}\right)-\frac{\tilde{V}}{2 \tilde{\phi}} \tilde{g}_{a b} .
\end{aligned}
$$

The stress-energy tensor $T_{a b}$ of matter, which by now we know is required to be conformally invariant if the operation (2.2), 2.3) is imposed to be a symmetry of the theory, transforms according to $\tilde{T}_{a b}=\Omega^{-2} T_{a b}$ [14, 21]. Then the first term in the right-hand side of Eq. (2.21) becomes $8 \pi \tilde{T}_{a b} / \tilde{\phi}$ and the form of this equation is the same of Eq. 2.18 before the transformation. We conclude that Eqs. (2.2) and (2.3) describe a symmetry of BransDicke theory in the presence of an arbitrary (regular) scalar field potential and of conformally invariant matter. Examples include the Maxwell field in four spacetime dimensions already mentioned in Sec. IB and a radiation fluid with equation of state $P=\rho / 3$.

The Brans-Dicke field $\phi$ couples to the trace of the energy-momentum tensor of ordinary matter (cf. Eq. (2.19) and only conformally invariant matter is covariantly conserved after a conformal transformation $g_{a b} \rightarrow \tilde{g}_{a b}=\Omega^{2} g_{a b}$. In fact, as is well known in scalar-tensor gravity, the covariant conservation equation $\nabla^{b} T_{a b}=0$ becomes [1, 17, 21]

$$
\tilde{\nabla}^{b} \tilde{T}_{a b}=-\tilde{T} \nabla_{a} \ln \Omega,
$$

and only $T=0$ (which occurs if and only if $\tilde{T}=0$ ) guarantees covariant conservation after the conformal rescaling.

\section{APPLICATION TO BRANS-DICKE COSMOLOGY}

We now apply the new solution-generating technique to spatially homogeneous and isotropic Brans-Dicke cosmology (see [17, 21] for reviews). In general, this symmetry is not a Noether symmetry 22] nor a Hojman symmetry [23]. There are indications that the symmetry does not survive Wheeler-DeWitt quantization in minisuperspace (at least in the spatially flat case) because quantum 
effects cause an anomalous symmetry breaking similar to that occurring in condensed matter systems [24]. This fact is, however, immaterial in the present work, which is confined to classical gravity. First we use power-law, and then exponential solutions as seeds. In both cases the line element is the FLRW one in comoving coordinates

$$
d s^{2}=-d t^{2}+S^{2}(t)\left(\frac{d r^{2}}{1-k r^{2}}+r^{2} d \Omega_{(2)}^{2}\right)
$$

where the curvature index $k$ is normalized to $0, \pm 1$ and $d \Omega_{(2)}^{2}=d \theta^{2}+\sin ^{2} \theta d \varphi^{2}$ is the line element on the unit 2 -sphere.

Before proceeding we note that, in the case $\omega=-1$ corresponding to the bosonic string theory 3], the wellknown duality of pre-big-bang cosmology [25]

$$
S \rightarrow \bar{S}=1 / S, \quad \phi \rightarrow \bar{\phi}=S^{6} \phi
$$

is not reproduced by, and is unrelated to, the symmetry (2.2), (2.3) that we study in our work.

\section{A. Power-law solutions}

We first consider vacuum Brans-Dicke theory with $V \equiv$ 0 and we look for power-law solutions of the form

$$
\begin{aligned}
& S(t)=S_{0} t^{p}, \\
& \phi(t)=\phi_{0} t^{q},
\end{aligned}
$$

where $S_{0}>0, \phi_{0}>0, p$, and $q$ are constants. Most of the known exact solutions of Brans-Dicke cosmology are of this form [17], which includes the Brans-Dicke dust solution [1], the O'Hanlon and Tupper family [26], and the Nariai family [27]. Here we consider vacuum solutions.

After the conformal transformation (2.2) with parameter $\alpha$, the line element reads

$$
d \widetilde{s}^{2}=-t^{2 \alpha q} d t^{2}+S_{0}^{2} t^{2(p+\alpha q)}\left(\frac{d r^{2}}{1-k r^{2}}+r^{2} d \Omega_{(2)}^{2}\right)
$$

where an irrelevant multiplicative constant has been dropped. We now introduce the new time coordinate $\tau$ defined by $d \tau=t^{\alpha q} d t$ for $q \neq 0$, or

$$
t=(\alpha q+1)^{\frac{1}{\alpha q+1}} \tau^{\frac{1}{\alpha q+1}}
$$

with the choice of a common origin for $t$ and $\tau$ and $\alpha \neq$ $-1 / q, 1 / 2$. The line element (3.5) is then written using this comoving time as

$$
d \tilde{s}^{2}=-d \tau^{2}+S_{0}^{2} \tau^{\frac{2(p+\alpha q)}{\alpha q+1}}\left(\frac{d r^{2}}{1-k r^{2}}+r^{2} d \Omega_{(2)}^{2}\right)
$$

while the new Brans-Dicke field (2.3) is

$$
\tilde{\phi}(\tau)=(\alpha q+1)^{\frac{q(1-2 \alpha)}{\alpha q+1}} \phi_{0}^{1-2 \alpha} \tau^{\frac{q(1-2 \alpha)}{\alpha q+1}} .
$$

One can write

$$
\tilde{S}(\tau)=S_{0} \tau^{\tilde{p}}, \quad \tilde{\phi}(\tau)=\tilde{\phi}_{0} \tau^{\tilde{q}},
$$

where

$$
\begin{aligned}
\tilde{p} & =\frac{p+\alpha q}{\alpha q+1}, \\
\tilde{q} & =\frac{q(1-2 \alpha)}{\alpha q+1}, \\
\tilde{\phi}_{0} & =(\alpha q+1)^{\frac{q(1-2 \alpha)}{\alpha q+1}} \phi_{0}^{1-2 \alpha} .
\end{aligned}
$$

As a special situation, we discuss the O'Hanlon and Tupper family of spatially flat solutions of vacuum BransDicke cosmology given, for $k=0$, by 26 .

$$
\begin{aligned}
& q_{ \pm}=\frac{1}{3 \omega+4}\left(\omega+1 \pm \sqrt{\frac{2 \omega+3}{3}}\right), \\
& p_{ \pm}=\frac{1 \mp \sqrt{3(2 \omega+3)}}{3 \omega+4},
\end{aligned}
$$

whose exponents satisfy the relation

$$
3 q_{ \pm}+p_{ \pm}=1
$$

Equations (3.10) and (3.11) give

$$
3 \tilde{q}+\tilde{p}=\frac{1-5 \alpha q}{\alpha q+1},
$$

which is, in general, different from unity, hence the new solution generated here is not of the O'Hanlon and Tupper form.

\section{B. Exponential solutions with linear potential}

Instead of power-law solutions, we now use exponential solutions of vacuum Brans-Dicke theory with a linear potential $V(\phi)=\Lambda \phi$, which amounts to introducing a cosmological constant in this theory. The spatially flat family of solutions

$$
\begin{aligned}
& S_{ \pm}(t)=S_{0} \exp \left[ \pm(\omega+1) \sqrt{\frac{2 \Lambda}{(2 \omega+3)(3 \omega+4)}} t\right] \\
& \phi_{ \pm}(t)=\phi_{0} \exp \left[ \pm \sqrt{\frac{2 \Lambda}{(2 \omega+3)(3 \omega+4)}} t\right]
\end{aligned}
$$

with $S_{0}, \phi_{0}$ constants, are well-known attractors in phase space [28 30]. By performing the conformal transformation (2.2) one obtains

$$
\begin{aligned}
d \tilde{s}^{2}= & \phi^{2 \alpha} d s^{2}=-\mathrm{e}^{ \pm 2 \alpha \sqrt{\frac{2 \Lambda}{(2 \omega+3)(3 \omega+4)}} t} d t^{2} \\
& +S_{0}^{2} \exp \left[ \pm 2(\omega+1+\alpha) \sqrt{\frac{2 \Lambda}{(2 \omega+3)(3 \omega+4)}} t\right] \\
& \cdot\left(d r^{2}+r^{2} d \Omega_{(2)}^{2}\right) .
\end{aligned}
$$


The comoving time in the tilded world is

$$
\tau=\frac{\mathrm{e}^{ \pm \alpha \sqrt{\frac{2 \Lambda}{(2 \omega+3)(3 \omega+4)}} t}}{ \pm \alpha \sqrt{\frac{2 \Lambda}{(2 \omega+3)(3 \omega+4)}}}+\text { const. }
$$

One must make sure that $t$ and $\tau$ have the same direction. By choosing the positive sign this property follows trivially and $\tau=0$ corresponds to $t \rightarrow-\infty$. If the negative sign is taken, one can choose the integration constant so that

$$
\tau=\frac{1}{\alpha} \sqrt{\frac{(2 \omega+3)(3 \omega+4)}{2 \Lambda}}\left(1-\mathrm{e}^{-\alpha \sqrt{\frac{2 \Lambda}{(2 \omega+3)(3 \omega+4)}} t}\right) .
$$

Consider first the solution with positive sign, which is rewritten as

$$
d \tilde{s}^{2}=-d \tau^{2}+\tilde{S}^{2}(\tau)\left(d r^{2}+r^{2} d \Omega_{(2)}^{2}\right),
$$

where

$$
\begin{aligned}
& \tilde{S}(\tau)=\tilde{S}_{0} \tau^{\frac{\omega+1+\alpha}{\alpha}} \equiv \tilde{S}_{0} \tau^{\tilde{p}}, \\
& \tilde{\phi}(\tau)=\phi^{1-2 \alpha}=\tilde{\phi}_{0} \tau^{\frac{1-2 \alpha}{\alpha}} \equiv \tilde{\phi}_{0} \tau^{\tilde{q}},
\end{aligned}
$$

where

$$
\begin{aligned}
& \tilde{S}_{0}=S_{0}\left[\alpha \sqrt{\frac{2 \Lambda}{(2 \omega+3)(3 \omega+4)}}\right]^{\frac{\omega+1+\alpha}{\alpha}}, \\
& \tilde{\phi}_{0}=\phi_{0}^{1-2 \alpha}\left(\alpha \sqrt{\frac{2 \Lambda}{(2 \omega+3)(3 \omega+4)}}\right)^{\frac{1-2 \alpha}{\alpha}},
\end{aligned}
$$

and

$$
3 \tilde{q}+\tilde{p}=\frac{\omega+4-5 \alpha}{\alpha}
$$

(which, in general, is not equal to 1). The scalar field potential is now, according to Eq. (2.11), of the powerlaw form

$$
\tilde{V}(\tilde{\phi})=\tilde{\phi}^{\frac{-4 \alpha}{1-2 \alpha}} \Lambda \phi=\Lambda \tilde{\phi}^{\frac{1-4 \alpha}{1-2 \alpha}} .
$$

Using Eq. (2.10), the scale factor is written in terms of the new Brans-Dicke coupling $\tilde{\omega}$ as

$$
\tilde{S}(\tau)=\tilde{S}_{0} \tau^{\frac{1-2 \alpha}{\alpha}[\tilde{\omega}(1-2 \alpha)+1-3 \alpha]} .
$$

In this case, the symmetry (2.2), (2.3) transforms an exponential solution into a power-law one corresponding to a different power-law potential.

By choosing the negative sign in Eq. (3.20), we have instead the line element (3.23) with

$$
S(\tau)=S_{0}\left(1-\alpha \sqrt{\frac{2 \Lambda}{(2 \omega+3)(3 \omega+4)}} \tau\right)^{\frac{\omega+1+\alpha}{\alpha}}
$$

but $\tau$ is now given by Eq. (3.21) and

$$
\tilde{\phi}(\tau)=\phi_{0}^{1-2 \alpha}\left(1-\alpha \sqrt{\frac{2 \Lambda}{(2 \omega+3)(3 \omega+4)}} \tau\right)^{\frac{1-2 \alpha}{\alpha}} .
$$

The scalar field potential is again (3.28).

\section{A NEW FAMILY OF SPHERICAL, TIME DEPENDENT SOLUTIONS}

In this section we use the symmetry transformation to generate a new time-dependent solution of Brans-Dicke theory from a static one used as a seed.

A spherically symmetric and time-dependent solution of Jordan frame vacuum Brans-Dicke theory, which is conformal to the Fonarev spacetime1 [32, 33], was found recently in [34]. The line element and Brans-Dicke field are

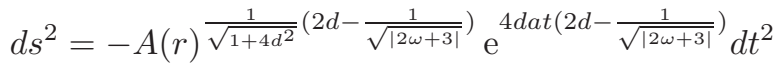

$$
\begin{aligned}
& +\mathrm{e}^{2 a t\left(1-\frac{2 d}{\sqrt{|2 \omega+3|}}\right)}\left[A(r)^{\frac{-1}{\sqrt{1+4 d^{2}}}\left(2 d+\frac{1}{\sqrt{|2 \omega+3|}}\right)} d r^{2}\right. \\
& \left.+A(r)^{1-\frac{1}{\sqrt{1+4 d^{2}}}\left(2 d+\frac{1}{\sqrt{|2 \omega+3|}}\right)} r^{2} d \Omega_{(2)}^{2}\right], \\
& \phi(t, r)=\phi_{0} \mathrm{e}^{\frac{4 d a t}{\sqrt{|2 \omega+3|}}} A(r)^{\frac{1}{\sqrt{|2 \omega+3|\left(1+4 d^{2}\right)}}},
\end{aligned}
$$

where

$$
\begin{gathered}
A(r)=1-\frac{2 m}{r}, \\
V(\phi)=V_{0} \phi^{\beta}, \quad \beta=2(1-d \sqrt{|2 \omega+3|}),
\end{gathered}
$$

and where $m>0, a, d$ are parameters of the family of solutions, while $\omega \neq-3 / 2$ and $\phi_{0}>0$ is another constant related to initial conditions.

We use a special case of this family as the seed to generate a new family of solutions of vacuum Brans-Dicke gravity. Assuming $a \neq 0$, the time dependence of the geometry (4.1) is eliminated if the parameter $d$ is simultaneously equal to $(2 \sqrt{|2 \omega+3|})^{-1}$ and to $\sqrt{|2 \omega+3|} / 2$, which is achieved if $\omega=-1$ or if $\omega=-2$. In these cases, however, the scalar field (4.2) remains time-dependent, while $\beta=1$ and the scalar field potential reduces to the linear $V(\phi)=V_{0} \phi$, which is equivalent to introducing a

\footnotetext{
1 The Fonarev solution of GR, in turn, is conformal to the FisherBuchdahl-Janis-Newman-Winicour-Wyman scalar field solution of the Einstein equations [31].
} 
cosmological constant in the Brans-Dicke action. Since $\phi>0$, this potential is effectively bounded from below. A scalar field which does not share the symmetries of the spacetime metric is currently of considerable interest because it is used in Brans-Dicke, Galileon, and Horndeski gravity as an ingredient to circumvent (35], see also [36]) well-known no-hair theorems for black holes [37 39].

Our starting point is

$$
\begin{aligned}
d s^{2} & =-d t^{2}+A(r)^{-\sqrt{2}} d r^{2}+A(r)^{1-\sqrt{2}} r^{2} d \Omega_{(2)}^{2}, \\
\phi(t, r) & =\phi_{0} \mathrm{e}^{2 a t} A(r)^{1 / \sqrt{2}} .
\end{aligned}
$$

This geometry is recognized as a special case of the Campanelli-Lousto geometry of Brans-Dicke theory. The general Campanelli-Lousto solution has the form [40]

$$
\begin{aligned}
d s_{C L}^{2}= & -A(r)^{b_{0}+1} d t^{2}+A(r)^{-a_{0}-1} d r^{2} \\
& +A(r)^{-a_{0}} r^{2} d \Omega_{(2)}^{2}, \\
\phi_{C L}(r)= & \phi_{0} A(r)^{\frac{a_{0}-b_{0}}{2}},
\end{aligned}
$$

where $a_{0}$ and $b_{0}$ are two parameters, only one of which is independent, and are related to the Brans-Dicke coupling by

$$
\omega\left(a_{0}, b_{0}\right)=\frac{-2\left(a_{0}^{2}+b_{0}^{2}-a_{0} b_{0}+a_{0}+b_{0}\right)}{\left(a_{0}-b_{0}\right)^{2}} .
$$

The line element (4.5) and scalar field (4.6) are reproduced if $\left(a_{0}, b_{0}\right)=(\sqrt{2}-1,-1)$, while Eq. (4.9) gives back $\omega=-1$ (but not the value $\omega=-2$ because the Campanelli-Lousto solution holds for $\omega>-3 / 2$ ). BransDicke gravity with this value of the Brans-Dicke coupling corresponds to the low-energy limit of bosonic string theory [3], so it is plausible that the spacetime (4.5), (4.6) has some stringy analogue. Although it was originally presented as describing a black hole spacetime, it was attributed a zero temperature, and there are studies of the thermodynamics of such "cold black holes" [41], the Campanelli-Lousto solutions can only describe wormholes or naked singularities but not black holes [42]. When the parameter $a_{0}$ is positive, and therefore for the value $a_{0}=\sqrt{2}-1$ corresponding to (4.5), (4.6), the Campanelli-Lousto geometry describes a wormhole with the throat located at the apparent horizon radius [42]

$$
r_{H}=\left(2+a_{0}\right) m=(\sqrt{2}+1) m,
$$

which corresponds to the value

$$
\begin{aligned}
R_{H} & =\left(2+a_{0}\right)^{\frac{a_{0}+2}{2}} a_{0}^{-a_{0} / 2} m \\
& =(\sqrt{2}+1)^{\frac{\sqrt{2}+1}{2}}(\sqrt{2}-1)^{\frac{1-\sqrt{2}}{2}} m
\end{aligned}
$$

of the areal radius $R(r)=r A(r)^{-a_{0} / 2}$. By applying the symmetry transformation (2.2) and (2.3), we obtain the new solution of vacuum Brans-Dicke theory with scalar field potential

$$
\tilde{V}(\tilde{\phi})=V_{0} \tilde{\phi}^{\frac{1-4 \alpha}{1-2 \alpha}}
$$

and Brans-Dicke coupling

$$
\tilde{\omega}=\frac{6 \alpha(1-\alpha)-1}{(1-2 \alpha)^{2}}
$$

given by

$$
\begin{aligned}
d \tilde{s}^{2}= & -\mathrm{e}^{4 \alpha a t} A(r)^{\alpha \sqrt{2}} d t^{2}+\mathrm{e}^{4 \alpha a t}\left[A(r)^{-\sqrt{2}(1-\alpha)} d r^{2}\right. \\
& \left.+A(r)^{1-\sqrt{2}(1-\alpha)} r^{2} d \Omega_{(2)}^{2}\right], \\
\tilde{\phi}(t, r)= & \tilde{\phi}_{0} \mathrm{e}^{2 a(1-2 \alpha) t} A(r)^{\frac{1-2 \alpha}{\sqrt{2}}}, \quad \tilde{\phi}_{0}=\phi_{0}^{1-2 \alpha} .
\end{aligned}
$$

(Incidentally, applying the symmetry transformation to the general Campanelli-Lousto spacetime (4.7), (4.8) does not produce another Campanelli-Lousto solution.) Equations (4.14) and (4.15) describe a 3-parameter family of solutions parametrized by $(m, a, \alpha)$. If $a=0$ the time dependence disappears and this solution reduces again to a Campanelli-Lousto geometry with new parameters $\left(a_{0}, b_{0}\right)=(\sqrt{2}(1-\alpha)-1, \alpha \sqrt{2}-1)$. Equation (4.9) then gives $\tilde{\omega}=\left(-6 \alpha^{2}+6 \alpha-1\right)(1-2 \alpha)^{-2}$ which, of course, matches Eq. 2.10 for $\omega=-1$.

If $a \neq 0$, the new time coordinate

$$
\tau=\frac{\mathrm{e}^{2 \alpha a t}}{2 \alpha a}
$$

transforms the spacetime $\left(\tilde{g}_{a b}, \tilde{\phi}\right)$ into

$$
\begin{aligned}
d \tilde{s}^{2}= & -A(r)^{\alpha \sqrt{2}} d \tau^{2}+(2 \alpha a \tau)^{2}\left[A(r)^{-\sqrt{2}(1-\alpha)} d r^{2}\right. \\
& \left.+A(r)^{1-\sqrt{2}(1-\alpha)} r^{2} d \Omega_{(2)}^{2}\right], \\
\tilde{\phi}(\tau, r)= & \phi_{*} \tau^{\frac{1-2 \alpha}{\alpha}} A(r)^{\frac{1-2 \alpha}{\sqrt{2}}}, \quad \tilde{\phi}_{*}=\left[(2 \alpha a)^{1 / \alpha} \phi_{0}\right]^{1-2 \alpha} .
\end{aligned}
$$

By taking the limit $m \rightarrow 0$, this geometry reduces to the spatially flat FRLW universe

$$
d \tilde{s}_{(1)}^{2}=-d \tau^{2}+(2 \alpha a \tau)^{2}\left(d r^{2}+r^{2} d \Omega_{(2)}^{2}\right)
$$

with comoving time $\tau$, linear scale factor $S(\tau)=2 \alpha a \tau$, and scalar field

$$
\tilde{\phi}_{(1)}(\tau, r)=\phi_{*} \tau^{\frac{1-2 \alpha}{\alpha}}
$$

(this is not a O'Hanlon and Tupper universe). The same line element and Brans-Dicke scalar are obtained asymptotically for $r \gg 2 m$. Therefore, the new solution is interpreted as a spherical inhomogeneity in a spatially flat 
FLRW universe, with the scalar $\phi$ behaving asymptotically as a perfect fluid with equation of state $P=-\rho / 3$. It is not trivial to establish the nature of the central inhomogeneity. Since the Campanelli-Lousto geometry with parameter $a_{0}>0$, to which the new solution is conformal, can describe only a wormhole [42], one would naively expect that its conformal cousin describes the same type of solutions. While this is indeed the case for spherical geometries resulting from the conformal transformation of a wormhole or a naked singularity with static conformal factor [43], a time-dependent conformal factor may change this picture. As an example, a timedependent conformal transformation of the static Fisher solution (which contains a naked singularity 31]), produces the Husain-Martinez-Nuñez solution of the Einstein equations in which a central singularity is covered by a black hole apparent horizon for part of the history of this spacetime (according to the comoving time of the FLRW background) [4]. A detailed study of the physical interpretation of the solution (4.17) and (4.18) will be reported elsewhere.

\section{GENERATING NEW AXIALLY SYMMETRIC SOLUTIONS}

The solution-generating technique can be applied to cylindrically symmetric spacetimes. Since the Brans Dicke action remains invariant when an electromagnetic field is added to it, we present a new solution generated by using a cylindrically symmetric electrovacuum Brans Dicke spacetime as a seed [45]. The latter contains only an azimuthal magnetic field $B$ and the line element takes the form

$$
\begin{aligned}
d s^{2}= & \left(1+c^{2} r^{p}\right)^{2}\left[r^{2(q-d)}\left(-d t^{2}+d r^{2}\right)\right. \\
& \left.+W_{0}^{2} r^{2(k-d)} d \theta^{2}\right]+\frac{r^{2 d}}{\left(1+c^{2} r^{p}\right)^{2}} d z^{2}
\end{aligned}
$$

in cylindrical coordinates $(t, r, \theta, z)$, where

$$
\begin{aligned}
& p=2 d-k+1 \\
& q(\omega)=d(d-k+1)+\frac{\omega}{2}(k-1)^{2}+k(k-1) .
\end{aligned}
$$

The scalar and the magnetic field are

$$
\begin{aligned}
& \phi(r)=\phi_{0} r^{1-k}, \\
& B_{r}=B_{z}=0, \quad B_{\theta}(r)= \pm \frac{\sqrt{\phi_{0}} c p r^{p-1}}{\left(1+c^{2} r^{p}\right)^{2}}
\end{aligned}
$$

respectively. $\phi_{0}$ is a positive constant, while the constant $c$ is related to the coupling of the electromagnetic field to the current [45]. If $c=0$, (5.1)-(5.5) becomes the electrovacuum solution of [46] with the Levi-Civita geometry. When $c=0$, the constant $W_{0}$ introduces a conical singularity (if $W_{0}^{2} \neq 1$ ), while the constant $d$ is related with the energy density of the electromagnetic source. For $d=0$, the line element (5.1) describes a cosmic string in Brans-Dicke-Maxwell theory in which the $z$-axis carries a current [47]. $k$ is a free parameter and, for $k=1$, this spacetime reduces to an Einstein-Maxwell solution [11, 48]. If $c=0$ and $k=1$ simultaneously, this geometry reduces to the Levi-Civita solution of the vacuum Einstein equations [49]. Equations (5.1)- (5.5) describe a family of solutions characterized by the four parameters $(\omega, k, d, c)$, where $\omega$ is a parameter of the theory.

After performing the conformal transformation (2.2), the line element (5.1) is

$$
\begin{aligned}
d \tilde{s}^{2}= & \left(1+c^{2} r^{p}\right)^{2}\left\{r^{2[q-d+\alpha(1-k)]}\left(-d t^{2}+d r^{2}\right)\right. \\
& \left.+W_{0}^{2} r^{2[k-d+\alpha(1-k)]} d \theta^{2}\right\}+\frac{r^{2[d+\alpha(1-k)]}}{\left(1+c^{2} r^{p}\right)^{2}} d z^{2}
\end{aligned}
$$

where $q$ is simply given by (5.3). In the conformally transformed frame, one can also express this equation in terms of new Brans-Dicke parameter $\tilde{\omega}$ and of $\tilde{q} \equiv q(\tilde{\omega})$. Then Eq. (5.6) becomes

$$
\begin{aligned}
d \tilde{s}^{2}= & \left(1+c^{2} r^{p}\right)^{2}\left\{r^{2\left[\tilde{q}+\alpha(\alpha-1)(k-1)^{2}(2 \tilde{\omega}+3)-d+\alpha(1-k)\right]}\right. \\
& \left.\cdot\left(-d t^{2}+d r^{2}\right)+W_{0}^{2} r^{2[k-d+\alpha(1-k)]} d \theta^{2}\right\} \\
& +\frac{r^{2[d+\alpha(1-k)]}}{\left(1+c^{2} r^{p}\right)^{2}} d z^{2}
\end{aligned}
$$

and the new scalar field reads

$$
\tilde{\phi}=\tilde{\phi}_{0} r^{(1-2 \alpha)(1-k)} \quad \text { with } \quad \tilde{\phi}_{0}=\phi_{0}^{1-2 \alpha} .
$$

We have a new family of solutions characterized by the five parameters $(\tilde{\omega}, \alpha, k, c, d)$ (where $\tilde{\omega}$ is a parameter of the theory).

A conical singularity is present in both geometries (5.1) and (5.6), but we can eliminate it in the case $c=0$. Choosing $k-d=1$ and $W_{0}^{2}=1$ in Eq. (5.1) reduces the line element to

$$
\begin{aligned}
d s^{2}= & \left(1+c^{2} r^{p}\right)^{2}\left\{r^{p^{2}(\omega+2)}\left(-d t^{2}+d r^{2}\right)+r^{2} d \theta^{2}\right\} \\
& +\frac{r^{2 p}}{\left(1+c^{2} r^{p}\right)^{2}} d z^{2}
\end{aligned}
$$

while the scalar field is $\phi(r)=\phi_{0} r^{-p}$ and $p=d=k-1$. In the limit $c \rightarrow 0$, the conical singularity disappears.

For the new solution (5.6), we suggest that $W_{0}^{2}=1$ and $k-d+\alpha(1-k)=1$, then the metric becomes

$$
\begin{aligned}
d \tilde{s}^{2}= & \left(1+c^{2} r^{p_{0}}\right)^{2}\left[r^{p_{0}^{2}(\tilde{\omega}+2)}\left(-d t^{2}+d r^{2}\right)+r^{2} d \theta^{2}\right] \\
& +\frac{r^{2 p_{0}}}{\left(1+c^{2} r p_{0}\right)^{2}} d z^{2}
\end{aligned}
$$


where now $\tilde{\phi}=\tilde{\phi}_{0} r^{-p_{0}}$ and $p_{0}=(k-1)(1-2 \alpha)$. Of course, Eq. (5.9) is obtained as the $\alpha \rightarrow 0$ limit of Eq. (5.10).

Let us discuss now the limit to GR. For both the seed spacetime and the new spacetime, the limit $k \rightarrow 1$ (which implies $\left.p_{0} \rightarrow 0\right)$ reproduces GR and, if we choose $W_{0}^{2} \neq 1$ and we rescale the coordinates according to

$$
\begin{aligned}
& t \rightarrow \bar{t}=\left(1+c^{2}\right) t, \\
& r \rightarrow \bar{r}=\left(1+c^{2}\right) r, \\
& z \rightarrow \bar{z}=\frac{z}{1+c^{2}},
\end{aligned}
$$

we obtain the cosmic string geometry

$$
d s^{2}=-d \bar{t}^{2}+d \bar{r}^{2}+d \bar{z}^{2}+W_{0}^{2} \bar{r}^{2} d \theta^{2}
$$

with zero magnetic field. Rescaling the coordinates is de facto equivalent to setting $c=0$, but it is not necessary to do this explicitly: the magnetic field is automatically killed by making the scalar field constant in the GR limit of the new solution (but not in the seed solution). The string (5.14) has linear energy density $\mu$ along the $\bar{z}$-axis, where $W_{0}^{2}=1-4 \mu$. If $W_{0}^{2}<1$, there is a deficit angle and $\mu>0$ : the length of a circumference of radius $\bar{r}$ circling the $\bar{z}$-axis is $2 \pi \bar{r} \sqrt{1-4 \mu}$. If instead $W_{0}^{2}>1$, there is an excess angle corresponding to $\mu<0$ [50, 51].

\section{VI. $f(R)$ GRAVITY}

$f(R)$ theories of gravity are described by the action

$$
S_{f(R)}=\int d^{4} x \sqrt{-g} f(R)+S^{(\text {matter })},
$$

where $f(R)$ is a nonlinear function of the Ricci scalar and $S^{(m a t t e r)}$ is the matter action. The action describing the gravitational sector is equivalent to that of a subclass of scalar-tensor gravity [7]. In fact, it can be shown that the gravitational action in (6.1) is equivalent to that of a Brans-Dicke theory with Brans-Dicke field $\phi=f^{\prime}(R)$, Brans-Dicke coupling $\omega=0$, and scalar field potential

$$
V(\phi)=\phi R-\left.f(R)\right|_{R=R(\phi)},
$$

where $R$ is to be understood as a function of the scalar degree of freedom $\phi=f^{\prime}(R)$. It is then natural to ask whether the 1-parameter symmetry group of transformations (2.2), (2.3) of Brans-Dicke theory generates symmetries of $f(R)$ gravity, $f(R) \rightarrow \tilde{f}(\tilde{R})$. Answering this question turns out to be complicated. First of all, in order to keep the equivalence between $\tilde{f}(\tilde{R})$ gravity and
Brans-Dicke theory, it must be

$$
\begin{aligned}
\tilde{\phi} & =\frac{d \tilde{f}}{d \tilde{R}}, \\
\tilde{\omega} & =0, \\
\tilde{V}(\tilde{\phi}) & =\tilde{\phi} \tilde{R}-\tilde{f}(\tilde{R}) .
\end{aligned}
$$

Equation (6.4) fixes the parameter $\alpha$ of the transformation to be $\alpha=1$ and, at best, a single symmetry transformation of the $f(R)$ theory exists and not an entire 1-parameter group. Assuming $\alpha=1$, Eq. (6.3) gives $\tilde{\phi}=\phi^{-1}$. It is more complicated to enforce Eq. (6.5). Using Eq. 6.3), the latter becomes

$$
\tilde{V}(\tilde{\phi})=\tilde{\phi} \tilde{R}-\tilde{f}(\tilde{R}) ;
$$

it must be

$$
\tilde{\phi}^{3}(\tilde{R}-\tilde{\phi} f(R))=\tilde{\phi} \tilde{R}-\tilde{f}(\tilde{R})
$$

if the function $\tilde{f}(\tilde{R})$ is going to be generated by the operation (2.2), (2.3) with $\alpha=1$. By remembering the transformation property of the Ricci scalar under the conformal transformation $g_{a b} \rightarrow \tilde{g}_{a b}=\phi^{2} g_{a b}$ corresponding to $\alpha=1$

$$
\tilde{R}=\frac{1}{\phi^{2}}\left(R-\frac{6 \square \phi}{\phi}\right)
$$

14 17 and using the field equation (2.19) in vacuo or electrovacuo $(T=0)$, one obtains

$$
\tilde{R}=\frac{6}{(2 \omega+3)\left[f^{\prime}(R)\right]^{2}}\left[\frac{2 f(R)}{f^{\prime}(R)}-R\right] .
$$

If this equation could be inverted to express $R=R(\tilde{R})$ explicitly, the result could then be substituted into Eq. (6.8), producing a nonlinear ordinary differential equation for the function $\tilde{f}(\tilde{R})$ satisfied by all $\tilde{f}(\tilde{R})$ theories generated by the Brans-Dicke symmetry. Moreover, one could then write down explicitly the form of the function $\tilde{f}(\tilde{R})$. In practice, these steps cannot be performed. The root of the problem lies in the fact that the potential (6.2) of the scalar $\phi$ of $f(R)$ gravity is not explicit (because one cannot invert explicitly the relation $\phi=f^{\prime}(R)$ in order to obtain $\left.R=R(\phi)\right)$. Thus, in general, the question of whether the Brans-Dicke symmetry (2.2), (2.3) generates a symmetry of $f(R)$ gravity cannot be answered. However, we can propose a special solution to this problem.

A solution is found for the special choice $f(R)=R^{n}$ of the function $f(R)$, which has been the subject of an extensive literature [56, 57]. In this case we have

$$
\phi=n R^{n-1}, \quad V(\phi)=(n-1) R^{n}, \quad \tilde{\phi}=\frac{R^{1-n}}{n},
$$


and Eq. (6.7) becomes

$$
\tilde{R} \frac{d \tilde{f}}{d \tilde{R}}-\tilde{f}(\tilde{R})=\frac{n-1}{n^{4}} R^{4-3 n} .
$$

In vacuo or electrovacuo we have

$$
\frac{\square \phi}{\phi}=\frac{1}{2 \omega+3}\left(\frac{2 f}{\phi}-R\right)
$$

and

$$
\tilde{R}=\frac{(2 \omega n+9 n-12)}{n^{3}(2 \omega+3)} R^{3-2 n},
$$

which leads to

$$
R=\left[\frac{n^{3}(2 \omega+3)}{2 \omega n+9 n-12} \tilde{R}\right]^{\frac{1}{3-2 n}} .
$$

Equation (6.7) can then be written as the first order ordinary differential equation for $\tilde{f}(\tilde{R})$

$$
\tilde{R} \frac{d \tilde{f}}{d \tilde{R}}-\tilde{f}(\tilde{R})-\mu \tilde{R}^{\frac{3 n-4}{2 n-3}}=0
$$

where

$$
\mu=\left[\frac{n^{3}(2 \omega+3)}{2 \omega n+9 n-12}\right]^{\frac{3 n-4}{2 n-3}}\left(\frac{n-1}{n^{4}}\right) .
$$

A solution is

$$
\begin{aligned}
\tilde{f}(\tilde{R}) & =\alpha \tilde{R}^{\tilde{n}}, \\
\tilde{n} & =\frac{3 n-4}{2 n-3}, \\
\alpha & =\left(\frac{2 n-3}{n-1}\right) \mu .
\end{aligned}
$$

General theoretical constraints on any $f(R)$ gravity theory [7] are $f^{\prime}>0$, which guarantees that the graviton carries positive kinetic energy, and $f^{\prime \prime} \geq 0$, which guarantees local stability [58]. For $f(R)=R^{n}$, these constraints imply $n \geq 1$, and here we discard the value $n=1$ corresponding to GR, in which case $\phi=$ const. and the symmetry (2.2), (2.3) degenerates. Applying these constraints to the theory described by $\tilde{f}(\tilde{R})$ (but not necessarily to the "seed" theory $f(R)=R^{n}$ ) implies $\tilde{n}>1$, which is compatible with the experimental constraint

$$
\tilde{n}-1=(-1.1 \pm 1.2) \cdot 10^{-5}
$$

coming from Solar System experiments [56].

For the special value $n=2$ of the exponent, and with vanishing Brans-Dicke parameter $\omega$, Eq. (6.18) implies that $\tilde{n}=2$ as well, while $\mu=\alpha=1$ and $\tilde{f}(\tilde{R})=\tilde{R}^{2}$. Therefore, the theory $f(R)=R^{2}$ is invariant under the transformation considered. Apart from the trivial case $n=1$ corresponding to GR, this is the only value of $n$ for which $\tilde{n}=n$. While this particular $f(R)$ model is inconsistent with weak gravity experiments, it constitutes a good approximation of the Starobinsky inflationary model of the early universe $f(R)=R+\alpha R^{2}[59]$ in strong curvature regimes.

Other possible solutions of Eq. (6.7) will be searched for in future work.

\section{CONCLUSIONS}

The symmetry group of a physical theory discloses some of its fundamental features. We have reported a symmetry of Brans-Dicke theory in vacuo, electrovacuo, and in the presence of conformally invariant matter, which includes a radiation fluid important in the radiation era of cosmology and in star models. This symmetry consists of a restricted conformal invariance of the theory under very specific conformal transformations accompanied by nonlinear redefinitions of the Brans-Dicke scalar, and it is not to be confused with the usual conformal transformation from the Jordan to the Einstein frame of scalar-tensor gravity and string theories. The symmetry was reported long ago [12] and was used to investigate anomalies [52] in the limit of Brans-Dicke gravity to GR ([12], see 53] for further developments). Here the symmetry of [12] is generalized to include the case in which the Brans-Dicke scalar $\phi$ is endowed with an arbitrary potential $V(\phi)$ and conformally invariant matter is possibly present.

We propose a novel use of this symmetry as a solutiongenerating technique, starting from a known solution of the theory used as a seed. As examples, we have reported new solutions of FLRW Brans-Dicke cosmology in the presence of a cosmological constant, a new 3parameter family of spherical, time-dependent vacuum solutions (which are rare in the literature, contrary to static spherical solutions which are much easier to find), and a new family of cylindrically symmetric static electrovacuum solutions. The new spherical family, which is achieved using an $\omega=-1$ solution as a seed and probably has stringy analogues, looks rather intriguing and its physical interpretation will be studied in more detail in the future. The new symmetry offers some scope for extending studies of point-like Lagrangians with cyclic variables in FLRW cosmology and in the realm of static spherical solutions, which have been investigated extensively in the literature in relation with Noether symmetries in scalar-tensor gravities (see 22 for a summary).

As pointed out long ago by Dicke [54], two conformal frames related by a conformal transformation are physically equivalent if the fundamental units of length, time, and mass (and all derived units) scale with appropriate powers of the conformal factor $\Omega$ of the transformation. In practice, it is not trivial to implement this requirement [42, [55]. This consideration is usually debated for the conformal transformation going from the Jordan to the Einstein conformal frame, which is excluded by our 
symmetry operation (2.2), (2.3), but Dicke's argument is more general and, therefore, a conformal transformation accompanied by the appropriate rescaling of units would not generate physically new solutions according to Dicke's argument. However, in this work we have not implemented Dicke's rescaling of units but we have used instead the transformation (2.2), (2.3) as a mathematical map. Therefore, the solutions obtained are indeed new solutions of the theory.

\section{ACKNOWLEDGMENTS}

We are grateful to Nathalie Deruelle for a discussion and to a referee for useful comments. D.K.C. thanks the
Scientific and Technological Research Council of Turkey (TÜBİTAK) for a postdoctoral fellowship through the Programme BIDEB-2219 and Namık Kemal University for support. V.F. is supported by the Natural Sciences and Engineering Research Council of Canada (grant number 2016-03803), and all authors thank Bishop's University.
[1] C.H. Brans and R.H. Dicke, Phys. Rev. 124, 925 (1961).

[2] P.G. Bergmann, Int. J. Theor. Phys. 1, 25 (1968); R.V. Wagoner, Phys. Rev. D 1, 3209 (1970); K. Nordvedt, Astrophys. J. 161, 1059 (1970).

[3] C.G. Callan, D. Friedan, E.J. Martinez, and M.J. Perry, Nucl. Phys. B 262, 593 (1985); E.S. Fradkin and A.A. Tseytlin, Nucl. Phys. B 261, 1 (1985).

[4] L. Amendola and S. Tsujikawa, Dark Energy, Theory and Observations (Cambridge University Press, Cambridge, UK, 2010).

[5] T. Clifton, P.G. Ferreira, A. Padilla, and C. Skordis, Phys. Rep. 513, 1 (2012); E. Berti et al., Class. Quantum Grav. 32, 243001 (2015); T. Baker, D. Psaltis, and C. Skordis, Astrophys. J. 802, 63(2015).

[6] S. Capozziello, S. Carloni, and A. Troisi, Rec. Res. Dev. Astron. Astrophys. 1, 625 (2003) arXiv:astro-ph/0303041; S.M. Carroll, V. Duvvuri, M. Trodden, and M.S. Turner, Phys. Rev. D 70, 043528 (2004).

[7] T.P. Sotiriou and V. Faraoni, Rev. Mod. Phys. 82, 451 (2010); A. De Felice and S. Tsujikawa, Living Rev. Relativity 13, 3 (2010); S. Nojiri and S.D. Odintsov, Phys. Rep. 505, 59 (2011).

[8] P.A.M. Dirac, Nature (London) 139, 323 (1937); Proc. Roy. Soc. Lon. A 165, 199 (1938); 333, 403 (1973).

[9] J.D. Barrow, The Constants of Nature (Pantheon Books, New York, 2002).

[10] H. Maeda and G. Giribet, J. High Energy Phys. 11 (2011) 015 .

[11] H. Stephani, D. Kramer, M. MacCallum, C. Hoenselaers, and E. Herlt, Exact Solutions of the Einstein Field Equations (Cambridge University Press, Cambridge, 2003).

[12] V. Faraoni, Phys. Lett. A 245, 26 (1998); Phys. Rev. D 59, 084021 (1999).

[13] J. Polchinski, String Theory (Cambridge University Press, Cambridge, 2005); M.B. Green, G.H. Schwarz, and E. Witten, Superstring Theory (Cambridge University Press, Cambridge, 1987).

[14] R.M. Wald, General Relativity (Chicago University Press, Chicago, 1984).

[15] J.L. Synge, Relativity: The General Theory (North Holland, Amsterdam, 1960).

[16] S.M. Carroll, Spacetime and Geometry: An Introduction to General Relativity (Addison Wesley, San Francisco, 2004).

[17] V. Faraoni, Cosmology in Scalar Tensor Gravity, Fundamental Theories of Physics Series vol. 139 (Kluwer Academic, Dordrecht, 2004).

[18] R.L. Seliger and G.B. Whitham, Proc. R. Soc. (London) A305, 1 (1968).

[19] B. Schutz, Phys. Rev. D 2, 2762 (1970).

[20] J.D. Brown, Class. Quantum Grav. 10, 1579 (1993).

[21] Y. Fujii and K. Maeda, The Scalar-Tensor Theory of Gravity (Cambridge University Press, Cambridge, 2003).

[22] S. Capozziello and V. Faraoni, Beyond Einstein Gravity (Springer, New York, 2010).

[23] S. Capozziello and M. Roshan, Phys. Lett. B 726, 471 (2013).

[24] S. Pal, Phys. Rev. D 94, 084023 (2016).

[25] G. Veneziano, Phys. Lett. B 265, 287 (1991); M. Gasperini and G. Veneziano, Phys. Lett. B 277, 256 (1992); A.A. Tseytlin and C. Vafa, Nucl. Phys. B 372, 443 (1992); A. Giveon, M. Porrati, and E. Rabinovici, Phys. Rep. 244, 77 (1994).

[26] J. O'Hanlon and B. Tupper, Nuovo Cimento B 7, 305 (1972).

[27] H. Nariai, Prog. Theor. Phys. 40, 49 (1968); L.E. Gurevich, A.M. Finkelstein, and V.A. Ruban, Astrophys. Sp. Sci. 22, 231 (1973).

[28] J.D. Barrow and K. Maeda, Nucl. Phys. B 341, 294 (1990).

[29] C. Romero and A. Barros, Gen. Rel. Grav. 25, 491 (1993).

[30] S.J. Kolitch, Ann. Phys. (NY) 246, 121 (1996).

[31] I.Z. Fisher, Zh. Eksp. Teor. Fiz. 18, 636 (1948) arXiv:gr-qc/9911008; O. Bergman and R. Leipnik, Phys. Rev. 107, 1157 (1957); A.I. Janis, E.T. Newman, and J. Winicour, Phys. Rev. Lett. 20, 878 (1968); H.A. Buchdahl, Int. J. Theor. Phys. 6, 407 (1972); M. Wyman, Phys. Rev. D 24, 839 (1981).

[32] O.A. Fonarev, Class. Quantum Grav. 12, 1739 (1995).

[33] H. Maeda, arXiv:0704.2731.

[34] V. Faraoni and S.D. Belknap-Keet, Phys. Rev. D 96, 044040 (2017).

[35] J.D. Bekenstein, in Second International A.D. Sahkarov Conference on Physics, Moscow, 20-24 May 1996 
(arXiv:9605059 [gr-qc]); T.P. Sotiriou and S.-Y. Zhou, Phys. Rev. Lett. 112, 251102 (2014); T.P. Sotiriou, Class. Quantum Grav. 32, 214002 (2015); C.A.R. Herdeiro and E. Radu, Int. J. Mod. Phys. D 24, 1542014 (2015).

[36] I. Rácz, Class. Quantum Grav. 16, 1695 (1999); Class. Quantum Grav. 18, 5103 (2001); A.A.H. Graham and R. Jha, Phys. Rev. D 90, 041501 (2014); B. Mesi and I. Smolić, arXiv:1407.7246; I. Smolić, Class. Quantum Grav. 32, 145010 (2015); F. Bagheri and R. Mansouri, arXiv:1408.0516 Y. Zhong, B.-M. Gu, S.-W. Wei, and Y.-X. Liu, Eur. Phys. J. C 76, 377 (2016); I. Smolić, Phys. Rev. D 95, 024016 (2017).

[37] S.W. Hawking, Commun. Math. Phys. 25, 167 (1972).

[38] T.P. Sotiriou and V. Faraoni, Phys. Rev. Lett. 108, 081103 (2012).

[39] S. Bhattacharya, K.F. Dialektopoulos, A.E. Romano, and T.N. Tomaras, Phys. Rev. Lett. 115, 181104 (2015).

[40] M. Campanelli and C. Lousto, Int. J. Mod. Phys. D 02, 451 (1993); C. Lousto and M. Campanelli, in The Origin of Structure in the Universe, Pont d'Oye, Belgium, 1992, edited by E. Gunzig and P. Nardone (Kluwer Academic, Dordrecht, 1993), p. 123.

[41] K.A. Bronnikov, M.S. Chernakova, J.C. Fabris, N. PintoNeto, and M.E. Rodrigues, Int. J. Mod. Phys. D 17 (2008) 25.

[42] L. Vanzo, S. Zerbini, and V. Faraoni, Phys. Rev. D 86, 084031 (2012).

[43] V. Faraoni, A. Prain, and A.F. Zambrano Moreno, Phys. Rev. D 93, 024005 (2016).

[44] V. Husain, E.A. Martinez, and D. Nuñez, Phys. Rev. D 50, 3783 (1994).

[45] A. Baykal and Ö. Delice, Gen. Relat. Gravit. 41, 267 (2009).

[46] A. Arazi and C. Simeone, Gen. Relat. Gravit. 32, 2259 (2000).

[47] A. Vilenkin and E.P.S. Shellard, Cosmic Strings and Other Topological Defects (Cambridge University Press, Cambridge, 1994).

[48] W.B. Bonnor, Proc. Phys. Soc. A 66, 145 (1953).
[49] T. Levi-Civita, Rend. Acc. Lincei 28, 101 (1919).

[50] M. Visser, Phys. Rev. D 39, 3182 (1989).

[51] G.E. Volovik, The Universe in a Helium Droplet (Clarendon Press, Oxford, 2003).

[52] N. Banerjee and S. Sen, Phys. Rev. D 56, 1334 (1997); T. Matsuda, Progr. Theor. Phys. 47, 738 (1972); C. Romero and A. Barros, Astrophys. Sp. Sci. 192, 263 (1992); Phys. Lett. A 173, 243 (1993); Gen. Relat. Gravit. 25, 491 (1993); F.M. Paiva and C. Romero, Gen. Relat. Gravit. 25, 1305 (1993); L.A. Anchordoqui, D.F. Torres, M.L. Trobo, and S.E. Perez-Bergliaffa, Phys. Rev. D 57, 829 (1998).

[53] B. Chauvineau, Class. Quantum Grav. 20, 2617 (2003); Gen. Relat. Gravit. 39, 297 (2007); L Järv, P. Kuusk, M. Saal, and O. Vilson, Class. Quantum Grav. 32, 235013 (2015).

[54] R.H. Dicke, Phys. Rev. 125, 2163 (1962).

[55] N. Deruelle and M. Sasaki, Springer Proc. Phys. 137, 247 (2011) arXiv:1007.3563.

[56] T. Clifton, Class. Quantum Grav. 23, 7445 (2006); J.D. Barrow and T. Clifton, Class. Quantum Grav. 23, L1 (2006); T. Clifton and J.D. Barrow, Phys. Rev. D 72, 103005 (2005); T. Clifton and J.D. Barrow, Class. Quantum Grav. 23, 2951 (2006); A.F. Zakharov, A.A. Nucita, F. De Paolis, and G. Ingrosso, Phys. Rev. D 74, 107101 (2006).

[57] A. Abebe, A. de la Cruz-Dombriz, and P.K.S. Dunsby, Phys. Rev. D 88, 044050 (2013); A. Abebe, M. Abdelwahab, A. de la Cruz-Dombriz, and P.K.S Dunsby, Class. Quantum Grav. 29, 135011 (2012); M. Aparicio Resco, A. de la Cruz-Dombriz, F.J. Llanes Estrada, and V. Zapatero Castrillo, Phys. Dark Universe 13, 147 (2016); A.V. Astashenok and S.D. Odintsov, Phys. Rev. D 94, 063008 (2016); P. Salucci, C. Frigerio Martins, and E. Karukes, Int. J. Mod. Phys. D 23, 1442005 (2014).

[58] V. Faraoni, Phys. Rev. D 74, 104017 (2006).

[59] A.A. Starobinsky, Phys. Lett. B 91, 99 (1980). 